\title{
P02.97. Lipoic acid supplementation induces a transient stress response and improves episodic memory and cholesterol efflux in humans
}

\author{
D Keith ${ }^{1 *}$, J Butler ${ }^{1}$, B Bemer ${ }^{1}$, J Mogle ${ }^{2}$, J Monette ${ }^{3}$, J Hair ${ }^{1}$, J Heinecke ${ }^{3}$, M Sliwinski ${ }^{2}$, T Hagen ${ }^{1}$ \\ From International Research Congress on Integrative Medicine and Health 2012 \\ Portland, Oregon, USA. 15-18 May 2012
}

\section{Purpose}

Lipoic acid (LA) shows promise as a beneficial micronutrient in improving health, particularly in the elderly. Clinical and in vitro reports show that LA induces endogenous antioxidants and acts as an anti-inflammatory agent. LA also increases nerve conductance, improves diabetes-induced polyneuropathies, and remediates the age-associated cognitive decline in canines. Furthermore, LA significantly improves hypertriglyceridemia and glucose handling. From our preclinical research we have found that LA primarily influences three areas of health: cognition, stress response, and lipids. This study examines the effects of LA on human subjects in components of each of the three aforementioned areas of health.

\section{Methods}

This study utilized acute treatments with LA supplements as well as chronic supplementation in an 8 week, doubleblind placebo-controlled cross-over trial in human volunteers. The subjects were grouped into young (18-45 years) or elderly ( $\sim 79$ years), and all subjects were administered the R-enantiomer of LA in the form of oral supplements. This was a small pilot study with 2-6 subjects in each experiment.

\section{Results}

Results from the backward letter span and paired associates cognitive tests indicate that LA improved verbal episodic memory in the elderly, but did not improve short-term memory in the young or elderly. Chronic LA altered stress response systems as indicated by a transient increase in salivary cortisol and aldehyde dehydrogenase 3A1. Chronic LA also increased the amount of cholesterol taken up by high-density lipoproteins, particularly in the elderly subjects.

\section{Conclusion}

LA may be useful as a complementary nutraceutical agent to improve cholesterol efflux and select memory processes, particularly in the elderly. Continuous supplementation with LA induces a transient catabolic state, as well as a transient stress response. Thus, LA may function as a hormetic agent by inducing an initial stress that primes the system to efficiently respond to future toxicological insult.

\section{Author details}

'Linus Pauling Institute, Oregon State University, Corvallis, USA. ${ }^{2}$ Pennsylvania State University, State College, USA. ${ }^{3}$ University of Washington, Seattle, USA.

Published: 12 June 2012

doi:10.1186/1472-6882-12-S1-P153

Cite this article as: Keith et al:: P02.97. Lipoic acid supplementation induces a transient stress response and improves episodic memory and cholesterol efflux in humans. BMC Complementary and Alternative Medicine 2012 12(Suppl 1):P153.

${ }^{1}$ Linus Pauling Institute, Oregon State University, Corvallis, USA

Full list of author information is available at the end of the article

(c) 2012 Keith et al; licensee BioMed Central Ltd. This is an Open Access article distributed under the terms of the Creative Commons Attribution License (http://creativecommons.org/licenses/by/2.0), which permits unrestricted use, distribution, and reproduction in any medium, provided the original work is properly cited. 